\title{
DNN-Based ADNMPC of an Industrial Pickling Cold-Rolled Titanium Process via Field Enhancement Heat Exchange
}

\author{
Biao Yang, ${ }^{1,2,3}$ Jinhui Peng, ${ }^{2,3}$ Wei Li, ${ }^{2,3}$ Youling Li, ${ }^{4}$ Huilong Luo, ${ }^{2,3}$ Zhuming Zhang, \\ Shenghui Guo, ${ }^{2,3}$ Shimin Zhang, ${ }^{2,3}$ Hezhou Su, ${ }^{4}$ and Yaming Shi ${ }^{4}$ \\ ${ }^{1}$ Faculty of Information Engineering and Automation, Kunming University of Science and Technology, \\ Kunming, Yunnan 650500, China \\ ${ }^{2}$ Faculty of Metallurgical and Energy Engineering, Kunming University of Science and Technology, Kunming, \\ Yunnan 650093, China \\ ${ }^{3}$ Key Laboratory of Unconventional Metallurgy, Ministry of Education, Kunming University of Science and Technology, \\ Kunming, Yunnan 650093, China \\ ${ }^{4}$ Kunming Iron \& Steel Holding Co., Ltd., Kunming, Yunnan 650302, China
}

Correspondence should be addressed to Jinhui Peng; jhpeng@kmust.edu.cn

Received 21 April 2015; Revised 31 July 2015; Accepted 16 August 2015

Academic Editor: Evangelos Tsotsas

Copyright (C) 2015 Biao Yang et al. This is an open access article distributed under the Creative Commons Attribution License, which permits unrestricted use, distribution, and reproduction in any medium, provided the original work is properly cited.

\begin{abstract}
The dynamic neural network based adaptive direct nonlinear model predictive control is designed to control an industrial microwave heating pickling cold-rolled titanium process. The identifier of the direct adaptive nonlinear model identification and the controller of the adaptive nonlinear model predictive control are designed based on series-parallel dynamic neural network training by RLS algorithm with variable incremental factor, gain, and forgetting factor. These identifier and controller are used to constitute intelligent controller for adjusting the temperature of microwave heating acid. The correctness of the controller structure, the convergence, and feasibility of the control algorithms is tested by system simulation. For a given point tracking, model mismatch simulation results show that the controller can be implemented on the system to track and overcome the mismatch system model. The control model can be achieved to track on pickling solution concentration and temperature of a given reference and overcome the disturbance.
\end{abstract}

\section{Introduction}

Pickling plates and strips are normally done in a continuous way, just by drawing the plates and strips from rolls through a cascade of picking tanks $[1,2]$. The idea behind pickling technologies was to enforce turbulences in the acid bath to reduce the pickling time to save chemical losses and to achieve the desired surface finish of metal products [3]. Pickling technologies for metal products are basically similar; only the pickling media are different. A number of investigations on pickling media have been reported [4-7], and considerable interest has been focused on environmentally friendly operations together with quality and cost. Pickling metal products are a hydrometallurgical processing consisting of mechanical descaling, electrochemical, and/or chemical operations [7]. The rate of pickling is affected by several variables, including the base metal product constituents, the type of adherence of oxides, time in the liquid bath, acid concentration, pickling bath temperature, and turbulence intensity $[2,8]$. Pickling rate increases with temperature and acid concentration [6]. The control of acid concentration has been studied by several investigators [9-12]. Kittisupakorn et al. [10] developed a neural network model predictive control to manipulate the hydrochloric acid concentration. The results show better performance over the conventional PI in the control of the nonlinear dynamic and multieffects baths. Daosud et al. [11] used neural network inverse model-based controller for the control acid concentration to be maintained at the optimum value. They reported the robustness of the proposed controller showing superiority when controlling such chemical manufacturing processes that have the distributed, highly nonlinear dynamic behavior, unmodeled dynamics, and dead 
time on inputs and measurements. Sohlberg [12] offered hybrid grey box model to control the complex pickling process. Literature, however, shows little investigation on the pickling bath temperature.

For decades, studies of pickling line have been mostly focused on the equipment design, material compositions, and process parameters design. It can be argued that the heat exchange will continue to play an important role in the foreseeable future in environmental management; especially today the concept of Sustainable Development recognizes that economic growth and environmental protection are inextricably linked [13]. The heating methods of the acid pickling solutions are classified into two types: direct steam mixed with the acid and indirect heating acid solution by using graphite heat exchanger [14]. There then followed several decades with no major advances until the use of the microwave irradiation of the acid liquid which is the transfer of electromagnetic energy to thermal energy and is energy conversion process, rather than heat transfer process of conventional heating. Microwave heating is unique and offers a number of advantages over conventional heating [15], and pilot plant investigation has indicated some advantages [16].

Although microwave heating has aforementioned advantages, to our knowledge, literature shows little on microwave irradiation acid pickling solution for pickling metal plates and strips. However, a recent pickling process using industrial microwave heating devices (IMHD) was developed for heating mixed acid (nitric acid and hydrofluoric acid) solution for pickling titanium plates and strips. Microwave powers generated by many magnetrons feed into the IMHD simultaneously; because distance between these input ports is very close to each other, electromagnetic mutual coupling phenomenon must take place between these input ports. Beyond that, it is well know that the temperature elevation inside a medium leads to changes in its physical properties and especially modifies the complex permittivity of the medium, As a result, the dissipated power, which depends on this quantity, becomes temperature-dependent. Depending on the microwave power, bistable steady-state temperatures may be expected. When changing the power, a switch from stable branch to another one may arise. The sudden increase of temperature controlled microwave heating, known as thermal runaway, has been studied since the phenomenon of runway could seriously damage the microwave heated object [17]. Microwave heating process is coupled with highly grossly nonlinear character and this depth of complexity is revealed in applications through the appearance of unusual and often unexpected physical behavior such as "hot sports" and "waiting time" phenomena $[18,19]$.

To solve the microwave heating acid solution for pickling the metal products, which, as described above in literature $[10-12,17]$, is a grossly nonlinear process of unmodeled dynamic, multivariable in nature interactions between baths, distributed processes, and uncertain and time-varying parameters which cause this process to be difficult to control by conventional controllers, it is necessary to model accurately nonlinear dynamical systems and to control efficiently strategy. However, obtaining accurate model for the steel pickling process and predicting its interacting and nonlinear behavior are actually highly difficult.

A recent approach to model and control nonlinear dynamical system is the use of neural networks (NN). The applications of NN for model identification and the adaptive control dynamic systems have been studied extensively [10, 20-22]. Recently, model predictive control (MPC) has been studied mostly as advanced control strategy which can handle most of the common process characteristics and industrial requirements in a satisfactory way. $\mathrm{NN}$ model-based process identification for use in MPC application has been reported $[10,23,24]$. The MPC methods reported in these papers all use the linearized form of the identified nonlinear NN model.

The objective of this investigation is first to construct on-line (dynamical) NN (DNN) identification model trained by the variable gain, variable forgetting factor, and resetting $[25,26]$ improved recursive least squares algorithm (RLSA) at each sampling instant and then to develop an adaptive direct nonlinear MPC (ADNMPC) algorithm based on LevenbergMarquardt with adaptive updating parameters [27], finally to compare the above DNN-ADNMPC with NN-based GPC algorithm using instantaneous linearization by solving a set of recursive Diophantine equations [28, 29]. The main difference between the DNN-ADNMPC and the GPC is that the former uses a nonlinear NN model to identify and control microwave heating pickling process directly whereas the latter utilizes a linearized form of a nonlinear NN model to identify and control microwave heating pickling process.

\section{Description of a Cold-Rolled Titanium Pickling Process}

The cold-rolled titanium plates and strips (Ti strips) pickling process consists of three major steps: prerinsing, pickling, and rinsing steps shown in Figure 1. The first step, namely, prerinsing bath containing $85^{\circ} \mathrm{C}$ by mixed acid of nitric acid (under $4 \%$ weight of $\mathrm{HNO}_{3}$ ) and hydrofluoric acid (under $0.8 \%$ weight of $\mathrm{HF}$ ), aims to remove the contaminants out of metal, also to preheat the metal and enhance the temperature of the metal before entering the first pickling bath (three pickling baths in all), and moreover to remove acid solution from the metal surface to avoid the pollution when being in the situation of metal move countercurrent to the acid solution. The purpose of the pickling step is to remove surface oxides (scales, namely, $\mathrm{TiO}_{2}, \mathrm{TiO}, \mathrm{Ti}_{2} \mathrm{O}_{3}$, and $\mathrm{TiO}_{3}$ ) on the $\mathrm{Ti}$ strips by an immersion of the metals into an aqueous acid solution. Ti strips are immersed in three pickling baths, containing $65^{\circ} \mathrm{C}, 60^{\circ} \mathrm{C}$, and $50^{\circ} \mathrm{C}$ of mixed acid solution of nitric acid, respectively, in order to remove the scales from the metals. The metals move countercurrently to the mixed acid stream. The reaction occurring in the pickling baths is as follows:

$$
\begin{aligned}
& \mathrm{TiO}_{X}+\mathrm{HNO}_{3}+\mathrm{HF} \longrightarrow \mathrm{Ti}\left(\mathrm{NO}_{3}\right)_{X}+\mathrm{TiF}_{X}+\mathrm{H}_{2} \mathrm{O} \\
&+\mathrm{NO} \uparrow
\end{aligned}
$$

Drag in-out of the mixed pickling solution of the three pickling baths is removed from the Ti strips surface using 


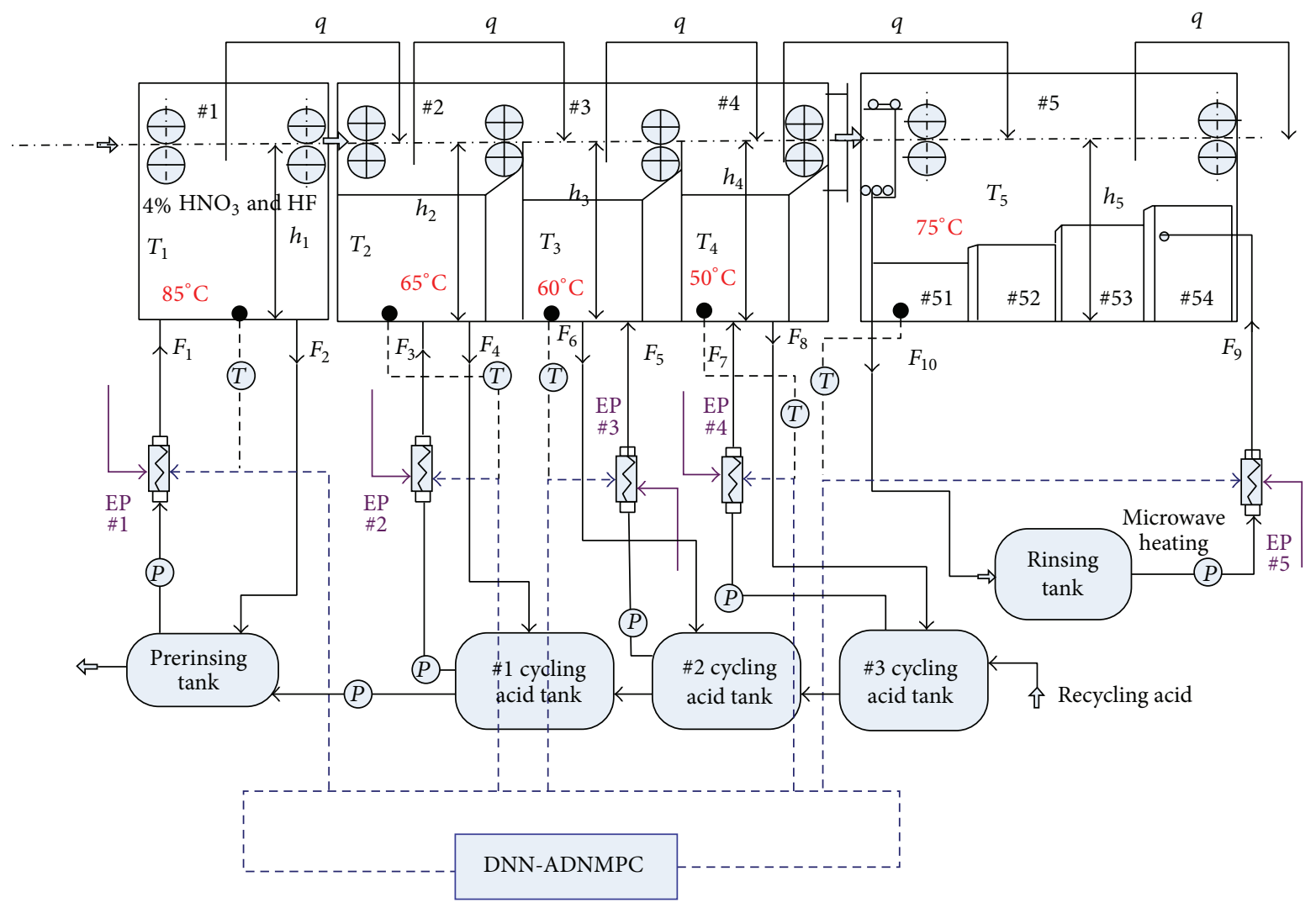

FIGURE 1: Simplified flow diagram of titanium pickling process control system.

rinsing water during the rinsing step, which consists of four $75^{\circ} \mathrm{C}$ desalted water baths. As shown in Figure 1, each rinsing stage of four baths connected in series has a separate circulatory system, respectively. The "new" water was pumped into \#4 bath and then there is progressive overflow into \#1 bath, which is opposite to the Ti strips move. In the above process rinsing bath, the "old" water was entered into the regenerated mixed acid tank. The main objective is to control temperature of each bath to a desired set point as illustrated in Figure 1, during the continuous acid pickling Ti strips by microwave heating the mixed acid $\left(\mathrm{HNO}_{3}\right.$ and $\left.\mathrm{HF}\right)$ solution. Mixed acid temperatures of prerinsing, the first pickling, the second pickling, the third pickling, and the rinsing baths are set at $85^{\circ} \mathrm{C}$, $65^{\circ} \mathrm{C}, 60^{\circ} \mathrm{C}, 50^{\circ} \mathrm{C}$, and $75^{\circ} \mathrm{C}$, respectively, by manipulating the electrical energy of the industrial microwave heating devices (IMHD).

Microwave generator (magnetron) is embedded in the IMHD to generate the necessary electric field strength $(E)$ for heating the mixed acid solution. The optimal $E$ is adjusted by the external electronic energy supplied magnetron, where their function is shown in (2); namely, the output power of microwave cavity heater of multiopen feed is shown as follows:

$$
n_{m} \cdot \phi U_{a} I_{a}=P_{i}, \quad(i=1, \ldots, 5),
$$

where $n_{m}$ is the number of the magnetrons, $\phi$ is the efficiency, $P_{i}$ is the microwave power of high frequency, and $U_{a} I_{a}$ is the input energy. Heat is then transported in the radial direction towards the centre of cavity of the IMHD and the mixed acid is heated. The internal architecture of the IMHD is shown in Figure 2.

A dynamic model of the continuous titanium pickling process, as shown in Figure 1, for the change in volume and temperature will be developed for all of the prerinsing, pickling, and rinsing steps based on the following assumptions:

(1) There is perfect mixing; thus, the exit temperature $T$ is also the temperature of the bath contents. And the heat of mixing is negligible compared to the heat of reaction.

(2) The density and heat capacity of the liquid are assumed to be constant. Thus, their temperature dependence is neglected.

(3) The concentration of pickling bath is assumed to be constant.

(4) The deterioration of bathing efficiency resulting from Titanium concentration is considered negligible.

(5) Heat losses to the ambient are negligible. 


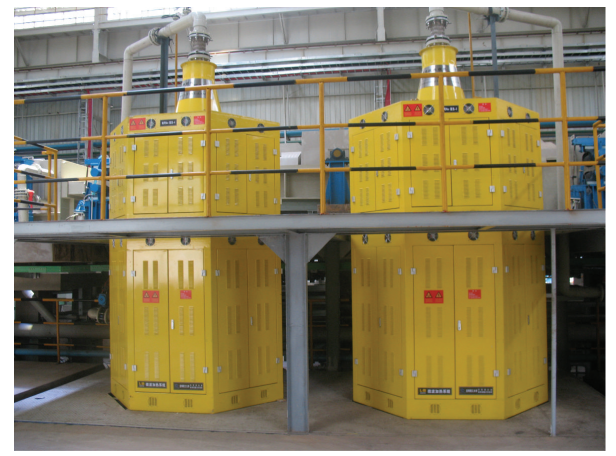

(a) Prototype of IMHD

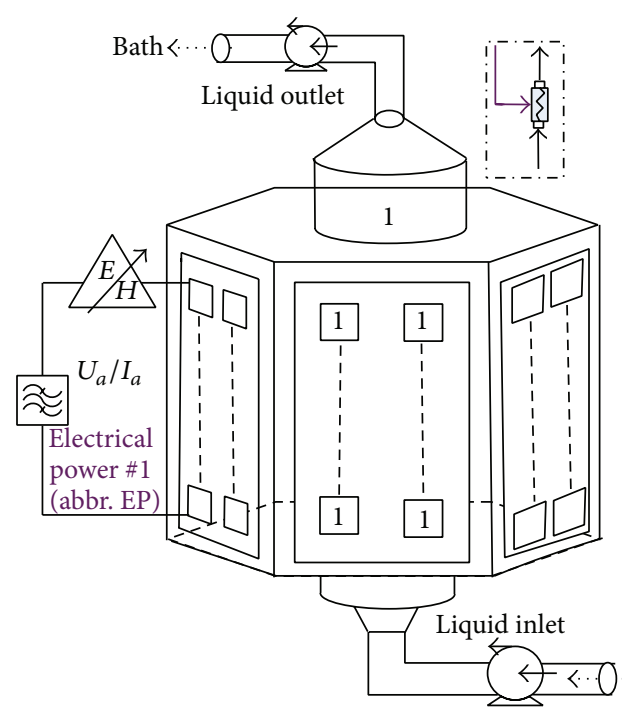

(b) Schematic of IMHD

Figure 2: The schematic diagram of the IMHD which is EP in Figure 1.

2.1. Prerinsing Step (the Temperature of $85^{\circ} \mathrm{C}$ and under Weight of $4 \% \mathrm{HNO}_{3}$ and $\mathrm{HF}$ Bath). Consider

$$
\begin{aligned}
\rho_{1} A \frac{d h_{1}}{d t}= & F_{1}-F_{2}-q, \\
V_{1} \rho_{1} C_{1} \frac{d T_{1}}{d t}= & C_{1}\left(F_{1}-F_{2}-q\right)\left(T_{\mathrm{sp}}-T_{1}\right) \\
& +\left(-\Delta H_{R}\right) V r_{1}+P_{1} .
\end{aligned}
$$

2.2. Pickling Step (the Temperature of $65^{\circ} \mathrm{C}, 60^{\circ} \mathrm{C}$, and $50^{\circ} \mathrm{C}$ $\mathrm{HNO}_{3}$ and $\mathrm{HF}$ Baths). Consider

$$
\begin{aligned}
\rho_{2} A \frac{d h_{2}}{d t}= & F_{3}-F_{4}, \\
\rho_{3} A \frac{d h_{2}}{d t}= & F_{5}-F_{6}, \\
\rho_{4} A \frac{d h_{2}}{d t}= & F_{7}-F_{8}, \\
V_{2} \rho_{2} C_{2} \frac{d T_{2}}{d t}= & C_{2}\left(F_{3}-F_{4}\right)\left(T_{\mathrm{sp}}-T_{2}\right) \\
& +q\left(C_{1} T_{1}-C_{2} T_{2}\right)+\left(-\Delta H_{R}\right) V r_{2} \\
& +P_{2},
\end{aligned}
$$

$$
\begin{aligned}
V_{3} \rho_{3} C_{3} \frac{d T_{3}}{d t}= & C_{3}\left(F_{5}-F_{6}\right)\left(T_{\mathrm{sp}}-T_{3}\right) \\
& +q\left(C_{2} T_{2}-C_{3} T_{4}\right)+\left(-\Delta H_{R}\right) V r_{3} \\
& +P_{3}, \\
V_{4} \rho_{4} C_{4} \frac{d T_{4}}{d t}= & C_{4}\left(F_{7}-F_{8}\right)\left(T_{\mathrm{sp}}-T_{4}\right) \\
& +q\left(C_{3} T_{3}-C_{4} T_{4}\right)+\left(-\Delta H_{R}\right) V r_{4} \\
& +P_{4} .
\end{aligned}
$$

2.3. Rinsing Step (the Temperature of $75^{\circ} \mathrm{C}$ in Four Desalted Water Baths). Consider

$$
\begin{aligned}
A \frac{d h_{5}}{d t}= & F_{9}-F_{10}, \\
V_{5} \rho_{5} C_{5} \frac{d T_{5}}{d t}= & C_{5}\left(F_{9}-F_{10}\right)\left(T_{\mathrm{sp}}-T_{5}\right) \\
& +q\left(C_{4} T_{4}-C_{5} T_{5}\right)+P_{5} .
\end{aligned}
$$

The rate of reaction (see (1)) is assumed to be first order with respect to the mixed acid of $\mathrm{HNO}_{3}$ and $\mathrm{HF}$, and considering the rate of reaction titanium unit volume $r_{i}$ as the function of reaction temperature $T_{i}$ is given by the Arrhenius relation as follows:

$$
r_{i}=k_{0} C_{\mathrm{ma}} \exp \left(-\frac{E_{\mathrm{ae}}}{R T_{i}}\right) \quad i=1, \ldots, 5,
$$


where $k_{0}$ is the frequency factor, $E_{\mathrm{ae}}$ is the activation energy, and $R$ is the molar gas constant.

In addition to the reaction $r$, the energy, $P$, transferred by the Microwave heat, needs to be imposed in order to complete the model of this continuous process. As pointed out in aforementioned section, microwave heating is the transfer of electromagnetic energy to thermal energy and is energy conversion, rather than heat transfer in conventional thermal processing. It is based on the power absorbed per unit volume that can be obtained from

$$
\begin{array}{r}
P_{i}=\sigma\left|E_{i}\right|^{2}=2 \pi f \varepsilon_{0} \varepsilon_{\mathrm{eff}}^{\prime \prime}\left|E_{i}\right|^{2}=2 \pi f \varepsilon_{0} \varepsilon_{r}^{\prime} \tan \delta\left|E_{i}\right|^{2}, \\
i=1, \ldots, 5,
\end{array}
$$

where $\varepsilon_{\text {eff }}^{\prime \prime}$ is the relative effective dielectric factor, $\varepsilon_{0}$ is the permittivity of free space, $f$ is the microwave frequency, $\sigma$ is the total effective conductivity, $\varepsilon_{r}^{\prime}$ is the relative dielectric constant, and $\tan \delta$ is the energy loss required to store a given quantity of energy. As can be seen from this equation, the dielectric properties $\left(\varepsilon_{\text {eff }}^{\prime \prime}, \varepsilon_{r}^{\prime}\right.$, and $\left.\tan \delta\right)$ assume a significant role in the extent of power absorbed by a material. The majority of the absorbed microwave power is converted to heat within the material, as shown in

$$
\frac{\Delta T}{\Delta t}=\frac{2 \pi f \varepsilon_{0} \varepsilon_{\mathrm{eff}}^{\prime \prime}|E|^{2}}{\rho C_{i}} .
$$

The dielectric properties $\left(\varepsilon_{\mathrm{eff}}^{\prime \prime}, \varepsilon_{r}^{\prime}\right.$, and $\left.\tan \delta\right)$ also are important parameters in determining the depth to which the microwaves will penetrate into the material. As can be seen by (9), the higher the values of $\varepsilon_{r}^{\prime}$ and $\tan \delta$, the smaller the depth of penetration for a specific wavelength:

$$
D=\frac{3 \lambda_{0}}{8.686 \pi \tan \delta\left(\varepsilon_{r}^{\prime} / \varepsilon_{0}\right)^{1 / 2}},
$$

where $D$ is the depth of penetration at which the incident power is reduced by one half and $\lambda_{0}$ is the incident wavelength. The depth of penetration is important since it will determine the uniformity of heating, curing, and so forth, throughout the material. High frequencies and large values of dielectric properties will result in surface heating, whereas low frequencies and small values of dielectric properties will result in more volumetric heating.

The process of pickling cold-rolled titanium plates and strips of acid baths by microwave irradiation is carefully depicted in this section. The variable gain, variable forgetting factor, and resetting RLS algorithm based DNN are used to predict the temperature of acid solution in prerinsing bath $\left(T_{1}\right)$, in three pickling baths $\left(T_{2}, T_{3}\right.$, and $\left.T_{4}\right)$, and in rinsing bath $\left(T_{5}\right)$, and ADNMPC strategy is utilized to adjust these temperatures to the desired set point by adjusting input electrical power of magnetrons $\left(U_{a}\right)$ as shown Figure 1 and (2). In the next section, the DNN and its training and validation are first introduced. Next, the DNN identification model is used to illustrate the ADNMPC strategy.

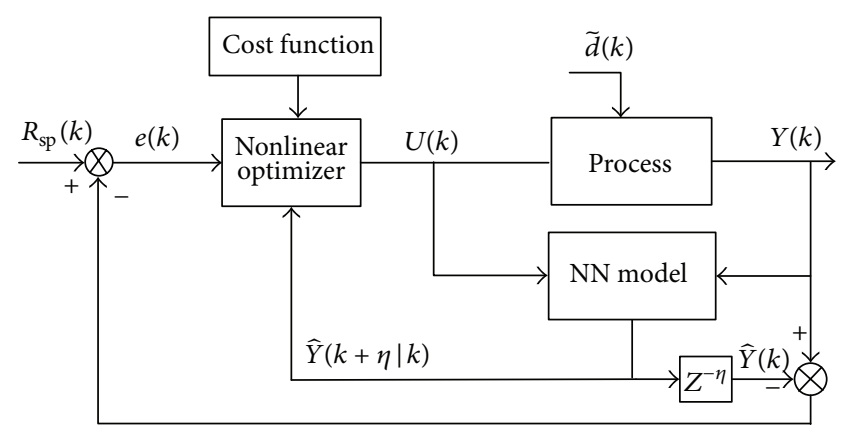

FIGURE 3: ADNMPC structure based on DNN for the pickling Ti strips process.

\section{DNN Model Identification and ADNMPC Control Strategy}

The proposed structure of DNN-based model identification and ADNMPC control strategies is shown in Figure 3.

In Figure 3, $k, R_{\mathrm{sp}}(k), e(k), U(k), Y(k), \widehat{Y}(k), \widehat{Y}(k+\eta \mid k)$, $\widetilde{d}(k)$, and $Z^{-\eta}$ are the number of samples, desired reference signal, prediction error, control input, process output, prediction output of the $\eta$ step delay, $\eta$ step ahead prediction output, disturbance, and $\eta$ step delay operator, respectively. NN model and nonlinear optimizer block represent the neural predictive models used to predict temperatures and the optimal manner used to obtain control input $U(k)$ in a specified prediction horizon.

3.1. Neural Network Modeling and Training. We propose an adaptive, namely, variable gain, variable forgetting factor, and resetting, recursive least squares (ARLS) algorithm for online NN training for nonlinear model identification using a series-parallel identification structure that is employed to approximate a nonlinear autoregressive moving average (NARMA) model structure through a minimization procedure. A class of nonlinear discrete-time multivariable plants can be described by the following NARMA model:

$$
\begin{gathered}
Y(k)=f(U(k-d), U(k-d-1), U(k-d-2), \ldots, \\
U(k-d-m), Y(k-1), Y(k-2), \ldots, Y(k-n)),
\end{gathered}
$$

where $f(U(\cdot), Y(\cdot))$ is a nonlinear function of its arguments and $d$ is the known time-delay of the system.

Given the input-output data pair $Z^{N}$ with known values of $m$ and $n$, it can be expressed in a more compact form of the output of system (10) as follows:

$$
Y(k)=f\left(Z^{N}, \varphi(k), \theta(k)\right)+\xi(k),
$$

where $Z^{N}=\{[U(N), Y(N)], N=1,2, \ldots\}$ is the input/output data of the system taken over NT period of time and where $N$ is the number of the data pairs and $T$ the sampling interval; $\varphi(k)=[U(k-d), \ldots, U(k-d-m), Y(k-1), \ldots, Y(k-n)]^{T}$ is the regression state vector, $\xi(k)$ is white noise vector, and $\theta(k)$ is unknown vector. 


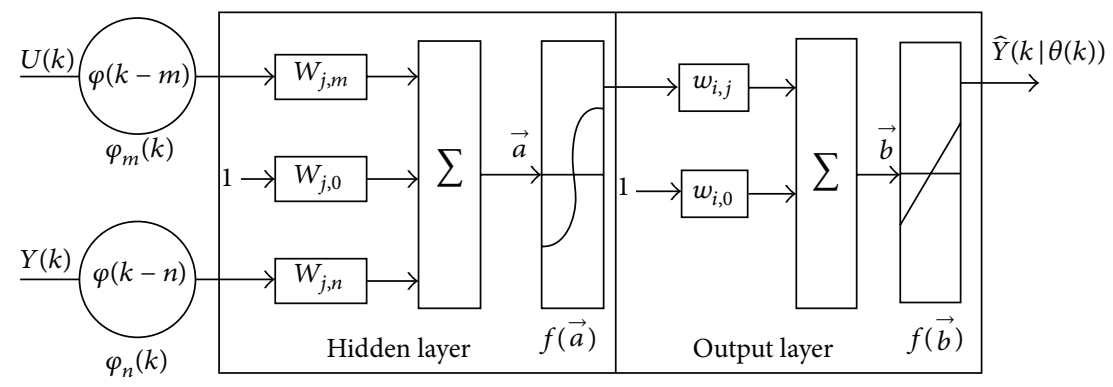

FIgURE 4: NN structure and its parameters.

The model identification problem is to construct a predictor that will produce an estimate of (11) provided that $m$ and $n$ are known; that is, $\widehat{Y}(k+1)=f\left(Z^{N}, \varphi(k), \theta(k)\right)$. At time $k+1$, once the $\theta(k+1)$ is obtained, the estimate of the output is given by $\widehat{Y}(k+1)=f\left(Z^{N}, \varphi(k), \theta(k+1)\right)$. So, it is obvious that the output estimates now depend on $\theta(k)$. Meanwhile, the purpose of the adaptive parameter identification algorithm is to estimate the true but unknown parameters $\theta(k)$ from the input-output data pairs; it is generally to adjust and update $\theta(k)$ recursively to obtain an optimal parameter $\hat{\theta}(k)$ satisfying an error function that measures the difference between the output of the NN and the output of the true system for given input data or input-output data pairs. Modeling based on $\mathrm{NN}$, obviously, is to update $\theta(k)$ recursively to obtain an optimal parameter $\hat{\theta}(k)$ by minimization of the following error estimates in the following form:

$$
\widehat{\theta}(k)=\arg \min \frac{1}{2 N} \sum_{k=1}^{N}[\varepsilon(k, \theta(k))]^{2},
$$

where $\widehat{Y}(k \mid \theta(k))=f\left(Z^{N}, \varphi(k), \theta(k)\right)$ is the general form of the predictor and defining $\varepsilon(k, \theta(k))=Y(k)-\widehat{Y}(k \mid \theta(k))$ for simplicity.

In general, there is no information other than that the bound $\bar{\varepsilon}_{k}$ on the unknown noise $\xi(k)$ is available; then, any estimate satisfying $|\varepsilon(k, \theta(k))|=|Y(k)-\widehat{Y}(k \mid \theta(k))| \leq \bar{\varepsilon}_{k}$ is compatible with the $Z^{k}=[U(k), Y(k)]$ at time $k$ and the noise bound $\varepsilon_{k}$. In application, to overcome this difficulty, that is, a noise bound must be available, we let $0<\varepsilon(1)<\varepsilon(2)<\cdots<$ $\varepsilon(s)$ be design variables called the tolerance levels and $s$ is the number of error levels. If $\left|\varepsilon_{k}(\theta(k))\right| \leq \varepsilon(k)$, a small gain is called for; if $\left|\varepsilon_{k}(\theta(k))\right| \geq \varepsilon(k)$, a large one is called for.

$\mathrm{NN}$ can approximate any nonlinear function to an arbitrary high degree of accuracy [30]. On the basis of its approximation capabilities, the $\mathrm{NN}$ technique is proposed for solving (12) and at the same time the convergence and stability of the network training in the presence of nonlinearities and uncertainties are guaranteed due to the continuously differentiable nature of the hidden layer activation function of the multilayer perceptron NN (MLPNN).

In system model identification the process to be modeled is in parallel with the NN model that will identify a model of the system (shown in Figure 3). The inputs to the NN are the past $m$-input and $n$-output samples contained in $\varphi(k)=\left[\varphi_{m}(k-m) \varphi_{n}(k-n)\right]$ obtained from $Z^{N}$ which denotes Tapped Delay Line memory used to store temporal NN input data. The NN considered in Figure 3 has a dynamic recurrent architecture and so it is called "Dynamic NN" because it incorporates temporary memory elements and feedback from the output of the system rather than the output of the NN model. It is the DNN guided on teacher forcing method that its output is forced to follow the system outputs on line in real-time.

The internal architecture of the proposed DNN model is a MLPNN with one hidden and one output layer as shown in Figure 4. Decomposing $\varphi(k)$ in (12) into the input and output parts as $\varphi_{m}(k)=[U(k-d), \ldots, U(k-d-m)]^{T}$ and $\varphi_{n}(k)=[Y(k-1), \ldots, Y(k-n)]^{T}$, respectively, the output of the DNN can be expressed in terms of the network parameters of Figure 4 as

$$
\begin{aligned}
\widehat{Y}(k \mid \theta(k))= & F_{i}\left(\sum_{j=1}^{N_{h}}\left(w_{i, j} f(\vec{a})+w_{i, 0}\right)\right), \\
\vec{a}= & \sum_{m=1}^{N_{m}} W_{j, m} \varphi_{m}(k) \\
& +\sum_{n=1}^{N_{n}} W_{j, n} \varphi_{n}(k-1)+W_{j, 0},
\end{aligned}
$$

where $j$ is the number of hidden nodes; $N_{m}$ and $N_{n}$ equal $m$ and $n$ in (10), respectively; $W_{j, m}$ and $W_{j, n}$ are the hidden and output weights, respectively; $W_{j, 0}$ and $w_{i, 0}$ are the hidden and output biases; $f_{j}(\vec{a})$ and $F_{i}(\vec{b})$ are an hyperbolic tangent activation function for the hidden layer and a linear activation function for the output layer, respectively. The former is defined as follows:

$$
f_{j}(\vec{a})=\frac{e^{\vec{a}}-e^{-\vec{a}}}{e^{\vec{a}}+e^{-\vec{a}}} .
$$

To get the optimal parameter $\hat{\theta}(k)$, we constitute $\theta(k)$ by the network weights $W_{j, m}, W_{j, n}$, and $W_{i, j}$ and biases or momentum $W_{j, 0}, W_{i, 0}$. It has been suggested in [30] that $\mathrm{NN}$ can be trained off-line by an already available data set $Z^{N}$ and the resulting network can be used for on-line adaptive controller design. The network training method is as follows. At time $k$, given $Z^{N}, \varphi(k)$, a small initial $\theta(k)=\theta_{0}$, and $U(k)$, $Y(k)$, the training algorithm computes the output estimate 
$\widehat{Y}(k+1 \mid \theta(k))$ using (13). At time $k+1$, according to the output of system $Y(k+1)$ and (12), it is used to adjust and update $\theta(k)$ until $\hat{\theta}(k)$ is obtained or a certain stopping criteria are satisfied, and $\hat{\theta}(k)=\theta(k+1)$; thus, the a posteriori output estimate $\widehat{Y}(k+1 \mid \theta(k+1))$ is available; $U(k+1), Y(k+1)$ is stored in Tapped Delay Line memory.

The proposed ARLS algorithm is based on the GaussNewton algorithm [31] with an adaptive updating rule based on the variable gain and variable forgetting factor and resetting $[25,26]$ is as follows.

Step 1. (a) Set $k=0$ and initialize $\theta(k)=\theta_{0}$; prepare $Z^{N}$, $\varphi(k)$, and current sample $U(k), Y(k)$.

(b) Compute $\widehat{Y}_{0}((k+1) \mid \theta(k))=f\left(Z^{N}, \varphi(k), \theta_{0}\right)$ using

Step 2. (a) Set $k=k+1$.

(b) Compute the changes in $\Delta \theta(k)$ given by the following formula:

$$
\Delta \theta(k)=\frac{\alpha_{k} P_{k-1} \varphi_{k}}{1+\varphi_{k}^{T} P_{k-1} \varphi_{k}} \varepsilon_{k}
$$

by using the recursive formulation of the modified RLS algorithm with an adaptive updating rule based on variable gain, variable forgetting factor, and resetting, where $P_{k}=$ $\left(1 / \lambda_{k}\right) P_{k-1}-\alpha_{k} P_{k-1} \varphi_{k} \varphi_{k}^{T} P_{k-1} /\left(1+\varphi_{k}^{T} P_{k-1} \varphi_{k}\right)+\beta I-\delta P_{k-1}^{2} ; \alpha_{k}$ is the adaptive algorithm gain; $\lambda_{k}$ is the adaptive algorithm forgetting factor. $\alpha_{k}, \beta, \delta$, and $\lambda_{k}$ are selected such that the following constraints are satisfied: $0<\gamma_{k}<\alpha_{k}<1, \beta>0$, $\delta>0$, and $\left(\gamma_{k}-\alpha_{k}\right)^{2}+4 \beta \delta<\left(1-\alpha_{k}\right)^{2}$ and $\sigma_{0} I<P_{0}<v_{0} I$, where $\gamma_{k} \equiv\left(1-\lambda_{k}\right) / \lambda_{k} ; v_{k} \equiv\left(\gamma_{k} / 2 \delta\right)\left[1+\left(1+4 \beta \delta / \gamma_{k}^{2}\right)^{1 / 2}\right] ; \sigma_{k}=$ $\left(\left(\alpha_{k}-\gamma_{k}\right) / 2 \delta\right)\left\{-1+\left[1+4 \beta \delta /\left(\alpha_{k}-\gamma_{k}\right)^{2}\right]^{1 / 2}\right\}$. Typically, $\alpha_{k}$ is the adjustable gain, $\alpha_{k} \in[0.1,0.65]$; $\beta$ is a small constant directly related to the minimum eigenvalue of $P(k), \beta \in[0,0.01] ; \lambda_{k}$ is the adjustable forgetting factor, $\lambda_{k} \in[0.9,0.99] ; \delta$ is a small constant that is inversely related to the maximum eigenvalue of $P(k), \delta \in[0,0.01]$

(c) Set $\theta(k)=\theta(k-1)+\Delta \theta(k)$.

(d) Compute $\widehat{Y}((k+1) \mid \theta(k))$ and $\varepsilon(k, \theta(k))=Y(k+1)-$ $\widehat{Y}(k+1 \mid \theta(k))$.

Step 3. Check if convergence, namely, $\hat{\theta}(k)$, has been achieved by using criterion of (12) based on updating $\theta(k)$ by checking $\varepsilon(k, \theta(k))$. If the criterion is satisfied, continue to Step 4; otherwise, go to Step 2.

Step 4. (a) Output $\theta^{*}(k)=\widehat{\theta}(k)$ and $\widehat{Y}^{*}((k) \mid \theta(k))=\widehat{Y}((k) \mid$ $\widehat{\theta}(k))$.

(b) Stop.

3.2. Validation of Trained NN. It has been suggested that $N N$ can be trained off-line by an already available data set, $Z^{N}$, and the resulting network can be used for on-line adaptive controller design. After training, the trained $\mathrm{NN}$ are validated by use of the $K$-step ahead validation $[22,32]$. In this method, $K$-step ahead predictions of the trained NN outputs are compared to the unscaled output training data. The following version is taken:

$$
\widehat{Y}(k+K \mid k, \widehat{\theta}(k))=f\left(Z^{N}, \widehat{\varphi}(k+K), \widehat{\theta}(k)\right),
$$

where $\widehat{\varphi}(k+K)=[U((k+K-1) \mid \widehat{\theta}(k)), \ldots, U((k+K-$ $m) \mid \hat{\theta}(k)), \widehat{Y}((k+K-1) \mid \hat{\theta}(k)), \ldots, \widehat{Y}((k+K-n) \mid \hat{\theta}(k))$, $Y((k+K-1) \mid \hat{\theta}(k)), \ldots, Y((k+K-n) \mid \widehat{\theta}(k))]^{T}$.

The mean value of the $K$-step ahead prediction error (MVPE) between the predicted output and the actual training data set is computed by

MVPE

$$
\begin{aligned}
& =\operatorname{mean}\left(\sum_{k=m+K}^{N} \frac{Y(k)-\widehat{Y}((k+K) \mid k, \widehat{\theta}(k))}{Y(k)}\right) \\
& \quad \times 100 \%,
\end{aligned}
$$

where $Y(k), \widehat{Y}((k+K) \mid k, \widehat{\theta}(k))$ correspond to the unscaled output training data and the $K$-step ahead predictive output, respectively. If the MVPE is not satisfied with predictionerror, the NN needs reconstruction; namely, update $\theta(k)$ by adjusting the weights $W_{j, m}, W_{j, n}$, and $W_{i, j}$ and biases $W_{j, 0}, W_{i, 0}$.

3.3. Adaptive Nonlinear Model Predictive Control Strategy. The proposed control strategy is dependent on an explicit process model and based on the receding horizon concept [33] shown in Figure 5. The GPC is a well-known class of MPC algorithms [34] based on the instantaneous linearization in an adaptive context by solving a set of recursive Diophantine equations and then applies quadratic programming to obtain the GPC control signal. The main difference between the GPC and the proposed ADNMPC is that the former uses a linearized version based on the instantaneous linearization of a nonlinear model, and in this control strategy the model identifier is itself controller, whereas the latter uses a nonlinear DNN model directly, being a nonlinear approximator and used in indirect adaptive control strategies as employed for ADNMPC design.

The deviations in system outputs, in the proposed NNbased identification and control scheme, (i) due to disturbances $\widetilde{d}(k)$ and/or (ii) model change due to a change in the operating conditions, as well as (iii) input changes because of the anticipated control inputs $U(k)$, are considered to correspond to the equivalent model changes, so that the identified NN model $\widehat{\theta}(k)$ in (12) is obtained on the basis of these equivalent model changes and the NNbased controller proposed is implemented on the basis of $\widehat{\theta}(k)$.

In the control strategy the $\mathrm{NN}$ mode is used to predict future temperature output, $\widehat{T}((k) \mid k, \widehat{\theta}(k)),\left(k=1,2, \ldots, N_{p}\right)$ over the prediction horizon $\left(N_{p}\right)$. The predicted outputs are passed to a nonlinear optimizer which produces the present and future control actions, $U_{a}(k)\left(k=1,2, \ldots, N_{u}\right)$ over the control horizon $\left(N_{u}\right)$ under condition of cost function satisfactorily. 


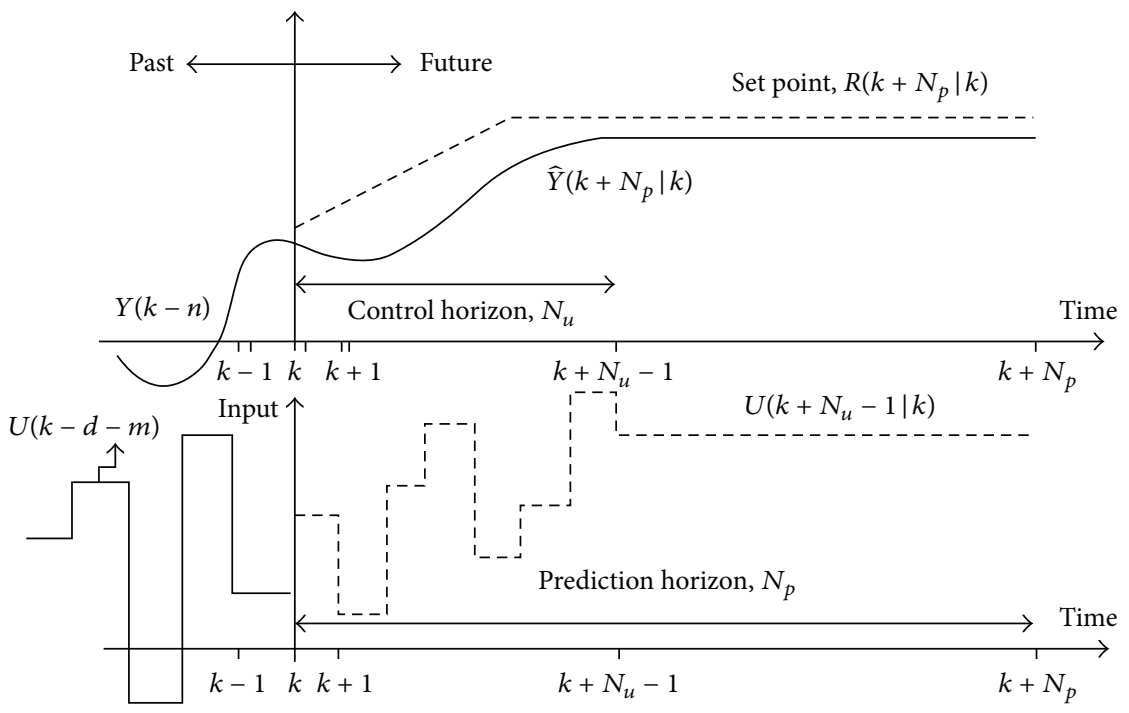

FIgURE 5: Predictive control: the basic idea.

A summary of adaptive nonlinear predictive control strategy, which is implemented in MATLAB and used to control the Ti strips pickling process simulation, is as follows:

(i) At the current sampling time $k$, sample the process outputs of bath temperature, $T_{i}(k), i=1, \ldots, 5$, and identify the NN model of pickling process.

(ii) Use the identified NN model (in Sections 3.1 and 3.2) to predict process output $\widehat{T}_{i}(k), \widehat{T}_{i}(k+$ $1), \ldots, \widehat{T}_{i}\left(k-1+N_{p}\right)$ corresponding to sampling time $k, k+1, \ldots, N_{p}$. (iii) Compute a sequence of manipulated variables $\widehat{U} a_{i}(k), \widehat{U} a_{i}(k+1), \ldots, \widehat{U} a_{i}\left(k-1+N_{u}\right)$, using a set of $\widehat{T}\left(k+N_{p} \mid k\right)$ to approach the desired set point in an nonlinear optimizer over a specified prediction horizon $N_{p}$. But the control input is to assumed to remain constant; thereafter, $\widehat{U} a_{i}\left(k+N_{u}\right)=\widehat{U} a_{i}(k+$ $\left.N_{u}+1\right)=\cdots=\widehat{U} a_{i}\left(k-1+N_{p}\right)$.

(iv) Apply nonlinear optimizer to obtain $\widehat{U} a_{i}(k)$ with $\widehat{T}\left(k+N_{p} \mid k\right)$ by minimizing the cost function of the below form

$$
\begin{aligned}
\text { Min } & \sum_{i=1}^{5} \sum_{\kappa=1}^{N_{p}}\left[W_{1}\left(T_{\text {tra }}(k+1)-\widehat{T}_{i}(k+\kappa)\right)^{2}+W_{2}\left(\widehat{U} a_{i}(k+\kappa) \widehat{U} a_{i}(k+\kappa)\right)\right] \\
\text { Subject to } & \widehat{U} a_{i}(\kappa)_{\min } \leq \widehat{U} a_{i}(k+\kappa) \leq \widehat{U} a_{i}(\kappa)_{\max }, \quad \kappa=1, \ldots, N_{p}, i=1, \ldots, 5, \\
& \widehat{T}_{i}(\kappa)_{\min } \leq \widehat{T}_{i}(k+\kappa) \leq \widehat{T}_{i}(\kappa)_{\max }, \\
& \Delta U a_{i}\left(k+N_{p}\right)=0, N_{u} \leq N_{p}-N_{d},
\end{aligned}
$$

where $W_{1}, W_{2}, N_{u}, N_{d}, N_{p}$, and $\Delta U a_{i}$ are weighting parameter penalizing change on output temperature $\widehat{T}_{i}(k)$, manipulated input $\widehat{U} a_{i}(k)$, maximum control horizon, minimum prediction horizon, maximum horizon, and the change in the manipulated input and $T_{\text {tra }}$ is the smoothing reference trajectory, defined here as

$$
\begin{array}{r}
T_{\text {tra }}(k+j)=\alpha^{j} T(k)+(1-\alpha)^{j} T_{\text {spi }}(k) \\
\quad\left(j=1,2, \ldots, N_{p}\right),
\end{array}
$$

where $T(k)$ and $\alpha$ are actual output temperature and smoothing factor, respectively.

(v) Computing the optimal control signals, implement the first a sequence of $N_{u}, \widehat{U} a_{i}(k), \widehat{U} a_{i}(k+$ $1), \ldots, \widehat{U} a_{i}\left(k-1+N_{u}\right)$ applied as the manipulated input signal to adjust the temperature of the pickling solution in the baths to the desired reference signal.

At each sample interval, although a sequence of $N_{u}$ future control moves (shown in (v)) is calculated by (18), only the first one $\widehat{U} a_{i}(k)$ is actually implemented and applied as the input signal to control the temperature of pickling solution; namely, $U a_{i}(k)=\widehat{U} a_{i}(k)$. The other elements are not applied 
in a receding horizon method, since a new output $T_{i}(k+1)$ is known based on new measurements at the next sampling interval $k=k+1$. Then the whole cycle of ADNMPC strategy of output measurement, prediction, and input signal determination is repeated, one sampling interval later: a new output measurement $T_{i}(k+1)$ is obtained; a new desired reference trajectory $T_{\mathrm{sp}_{i}}(k+i \mid k+1),(i=2,3, \ldots)$ is defined; predictions are made over the horizon $k+1+i$, with $i=1,2, \ldots, N_{p}$; a new input trajectory $\widehat{U} a_{i}(k+1+i \mid k+1)$ with $i=1,2, \ldots, N_{u}$ is chosen; and finally the next input is applied to the acid pickling process: $U a_{i}(k+1)=\widehat{U} a_{i}(k+1 \mid k+1)$.

\section{Simulation Results and Discussion}

The manipulated variables for the control of the pickling process are the microwave energy setting which regulates the electrical energy $U a_{i}(k)(i=1,2,3,4,5)$ and the flow rate of acid pickling medium to the microwave heating cavity $F_{j}(k)(j=1,3,5,7,9)$; namely, $U(k)=\left[U a_{i}(k) F_{j}(k)\right]^{\mathrm{T}}$. The controlled outputs of acid pickling process are the temperatures of the five baths, that is, prerinsing bath $T_{1}(k)$, first pickling bath $T_{2}(k)$, second pickling bath $T_{3}(k)$, third pickling bath $T_{4}(k)$, and rinsing bath $T_{5}(k)$, given by $Y(k)=$ $\left[T_{i}(k)\right] i=1, \ldots, 5$. The DNN-ADNMPC strategy is applied to control $Y(k)=\left[T_{i}(k)\right]$ to the normal values of $85^{\circ} \mathrm{C}, 65^{\circ} \mathrm{C}$, $60^{\circ} \mathrm{C}, 50^{\circ} \mathrm{C}$, and $75^{\circ} \mathrm{C}$ by adjusting $U(k)=\left[U a_{i}(k) F_{j}(k)\right]^{\mathrm{T}}$, respectively.

4.1. Identification of the NN Model of the Pickling Process. To develop a NN model to accurately model the acid pickling Ti strips process, the heat supplied from IMHD $(22 \mathrm{~kW})$ is varied by $\pm 10 \%$ (lower and upper values of IMHD are $19 \mathrm{~kW}$ and $24 \mathrm{~kW}$, resp.) in order to cover the entire operating range of the pilot plant, during both initial heat-up and acid pickling and to account for the possible uncertainties in the process model outside the operating region. Using these values of IMHD, an open-loop simulation of the acid pickling process based on its validated model of differential equations (2)-(6) obtained from first principles was performed with a sampling interval of $1 \mathrm{~min}$ to obtain input-output data pairs for the NN training, while these validation pairs were obtained from the actual acid pickling process.

The training data is scaled in order to prevent signals of largest magnitudes from dominating the identified model. Raw process data generated are scaled down to between 0.05 and 0.95 to avoid obtaining zero outputs and an infinite gain network. The network was trained for 80 epochs with the selected parameters: $K=3, N=6$, and $m=2$, and adaptive updating parameters of the exponential forgetting and resetting algorithms: $\alpha=0.6, \beta=0.01, \lambda=0.98$, and $\delta=0.01$.

After training, the weights of NN were rescaled so that the trained optimum structures can work with the unscaled data collected directly from the acid pickling process. The convergence of trained $\mathrm{NN}$ for the acid pickling process is shown in Figure 6. The fast convergence of the NN indicates that it is suitable for on-line dynamic nonlinear model identification. Next the trained NN was validated by using

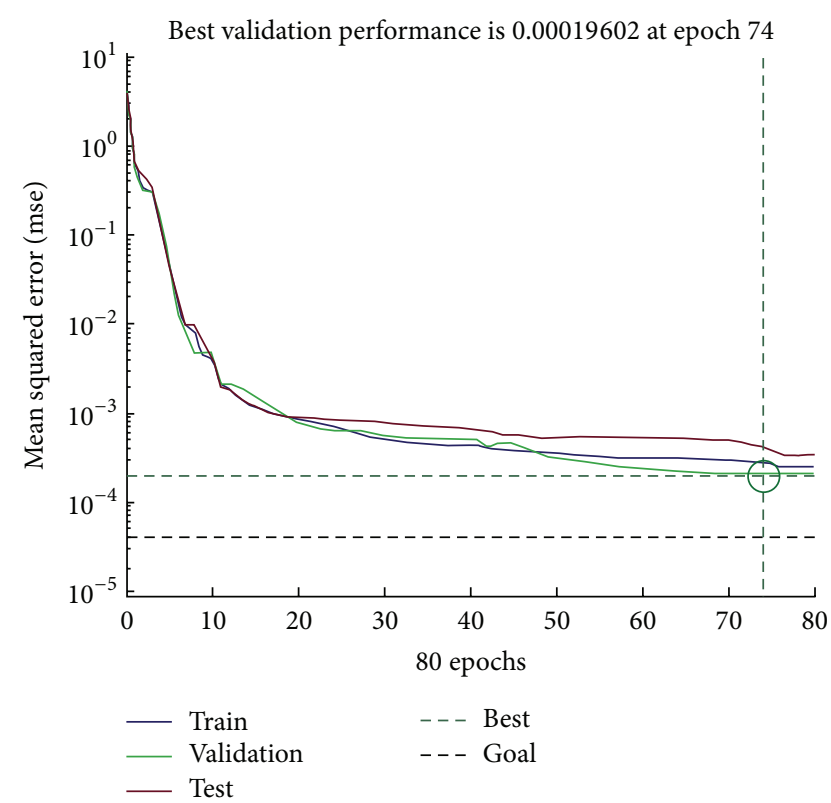

FIgURE 6: Convergence of adaptive dynamical NN for 80 epochs for the pickling process.

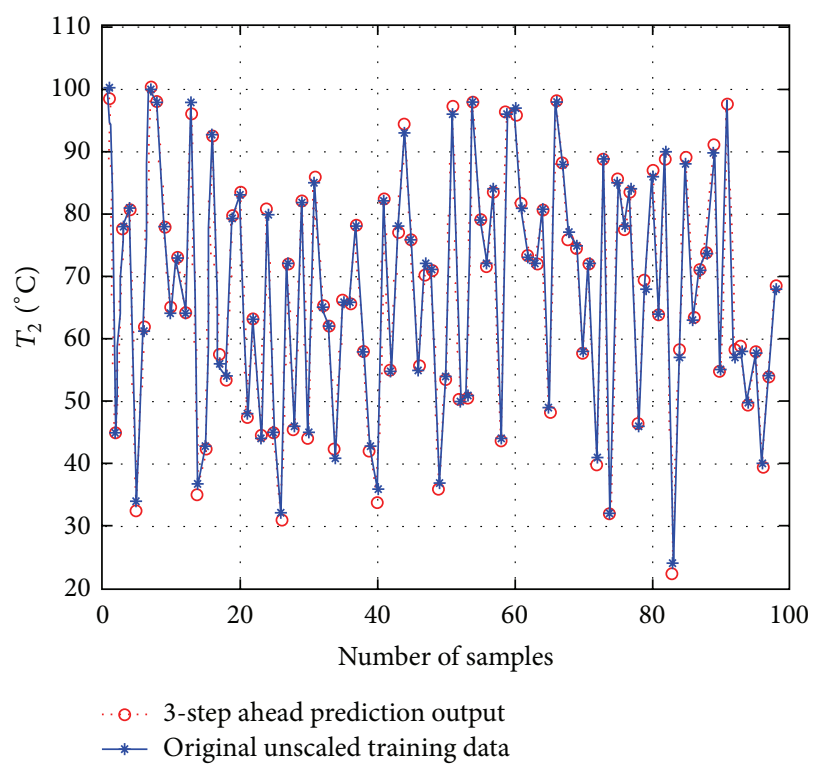

FIgURE 7: Comparison of the unscaled trained data (blue solid line) with 3-step ahead output predictions by the trained NN (red dotted line) for $\widehat{y}\left(T_{2}\right)$.

the 3-step ahead output predictions (explained in Section 3.2). The results shown in Figure 7 with red dotted lines for $T_{i}(k)$ were compared with the unscaled training data shown by the blue solid line. It can be seen that the predicted output follow very well the unscaled training data. Meanwhile, the MVPE calculated by (17) gives $0.640058 \%$ for $T_{i}(k)$ (shown in Figure 8). The values of small MVPE reveal that the identified NN model approximates the dynamics of the acid pickling process to a high degree of accuracy.

4.2. Control of the Pickling Process. The main control objective is to ensure that there is no overshoot in temperatures of 
TABLE 1: Parameters of DNN-ADNMPC control strategies.

\begin{tabular}{lccccc}
\hline Case & $\begin{array}{c}\text { Prediction horizon } \\
\left(N_{p}\right)\end{array}$ & Control horizon $\left(N_{u}\right)$ & $\begin{array}{c}\text { Weighting change on } \\
\text { output }\left(W_{1}\right)\end{array}$ & $\begin{array}{c}\text { Weighting change on } \\
\text { manipulated input } \\
\left(W_{2}\right)\end{array}$ & $\begin{array}{c}\text { Smoothing } \\
\text { factor }(\alpha)\end{array}$ \\
\hline Normal & 3 & 2 & 0.9 & 0.8 & 0.35 \\
Comp1 & 3 & 1 & 0.9 & 0.8 & 0.35 \\
Comp2 & 3 & 2 & 0.9 & 0.8 & 0.6 \\
\hline
\end{tabular}

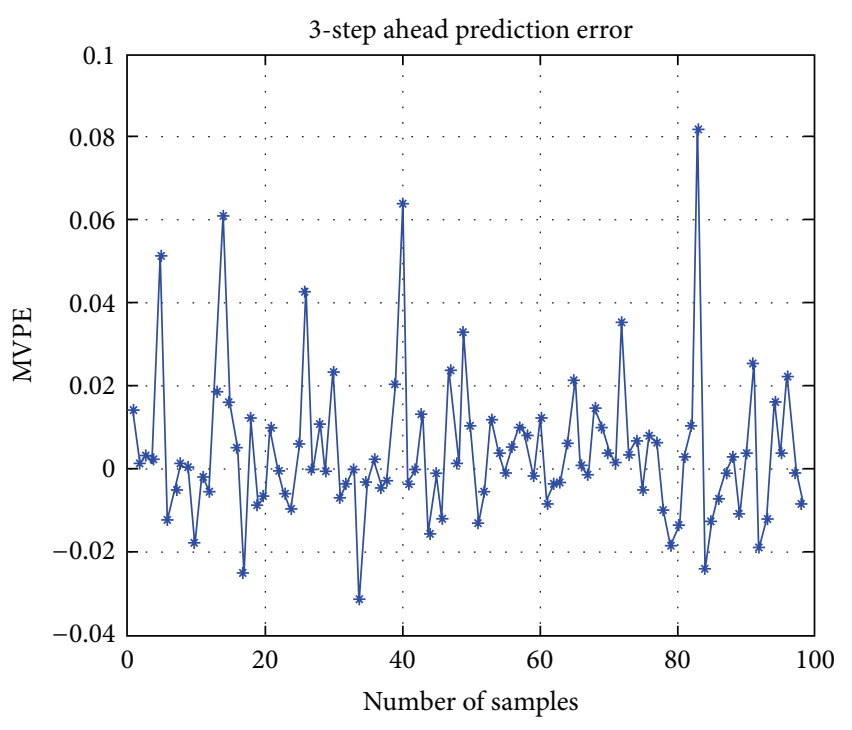

FIGURE 8: Values of MVPE.

the whole process of acid pickling Ti strips moving through prerinsing bath $\left(85^{\circ} \mathrm{C}\right)$, first pickling bath $\left(65^{\circ} \mathrm{C}\right)$, second pickling bath $\left(60^{\circ} \mathrm{C}\right)$, third pickling bath $\left(55^{\circ} \mathrm{C}\right)$, and rinsing bath $\left(75^{\circ} \mathrm{C}\right)$ sequentially, as a relatively small overshoot being outside of $\pm 2 \%$ might result in unacceptable surface topography and surface roughness [35].

According to previous research work, $[10,11]$ the simulations are divided into cases of control studies, (i) the set point tracking, (ii) disturbance case, (iii) model mismatch, and (iv) noise case. However, the present paper is focusing on (i) and (iii) because a change in the operating conditions (including input changes due to the anticipated control inputs, (ii) and (iv)) is considered to correspond to equivalent model changes.

The initially trained NN in Section 4.1 is used to tune the controller in an open-loop simulation. Next, the DNNADNMPC control strategies are programmed to run in closedloop with the first principles ((2)-(6)) model in the MATLAB environment, and set the parameters of DNNADNMPC control strategies in the Table 1 (Normal row). For the set point tracking case, their control performance for the temperature $T_{i}(k)$ output predictions is shown in Figure 9(a), while the manipulated input, namely, $U a_{i}(k)$, is shown in Figure 9(b). In this simulation, we allow the constraints on the maximum predicted temperature outputs to be $100^{\circ} \mathrm{C}$. As can be seen in Figure 9, the DNNADNMPC shows better control preference over the GPC. The GPC exhibits a small overshoot occasionally before tracking the desired reference signals, while in certain instances it cannot track the desired reference, as shown in Figure 9(a). Relatively, similar results are obtained in the prerinsing bath $\left(85^{\circ} \mathrm{C}\right)$, first pickling bath $\left(65^{\circ} \mathrm{C}\right)$, second pickling bath $\left(60^{\circ} \mathrm{C}\right)$, third pickling bath $\left(55^{\circ} \mathrm{C}\right)$, and rinsing bath $\left(75^{\circ} \mathrm{C}\right)$ and are not shown here.

For the model mismatch case, the rate of reaction in the pickling bath is considered as the model mismatch in parameter. The simulation of model mismatch is introduced by (i) (model-order change) changing the kinetic rate constant from its value by the first pickling bath $\left(65^{\circ} \mathrm{C}\right)$ and (ii) timelag change. Figure 10 shows the results of the DNNADNMPC and GPC control in this case. The figures illustrate that the DNNADNMPC strategy brought the temperatures to the set points by gradual increase of the magnetron voltage $U_{a 2}$ which give smooth manipulated response. The GPC control in turn brought the temperature to the set point by rigorous adjustment of the $U_{a 2}$ magnetron voltage causing overshoot and even instability in the pickling process with a long response time. Relatively, similar results are obtained for the prerinsing bath $\left(85^{\circ} \mathrm{C}\right)$, first pickling bath $\left(65^{\circ} \mathrm{C}\right)$, second pickling bath $\left(60^{\circ} \mathrm{C}\right)$, third pickling bath $\left(55^{\circ} \mathrm{C}\right)$, and rinsing bath $\left(75^{\circ} \mathrm{C}\right)$ and are not shown here. It indicates that DNNADNMPC gives less error and gives better control performances than the GPC controllers.

In addition, as can be seen in Figure 11 and Table 1, the performance of the identification and control using DNNADMPC strategy became worse with increasing of smoothing factor (Compl row in Table 1), and became better with of the increasing of the control horizons (Comp2 row in Table 1). From Figure 11 and Table 1, it can also be seen that the control actions were increased with decreasing the control horizon and decreased with increasing of the smoothing factor. Generally, control horizon $N=2$ is to meet requirement, and the extra computation time will be spent with increasing of $N_{u}$.

4.3. Computation Time for DNN Model Identification and Control of the Pickling Process. The DNN identification scheme and GPC and ADNMPC control strategies above were implemented on an Intel Core $1 \mathrm{CPU}$ running at $1400 \mathrm{MHz}$ using in the MATLAB Distributed and Parallel Toolbox in the MATLAB10. This MATLAB facility allows the utilization of the two processors available on the computer for the implementation of the identification and control algorithm at each time step. The sampling time of the 


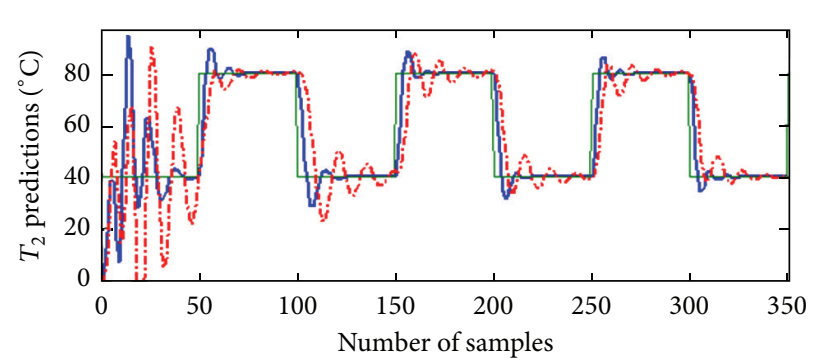

(a)

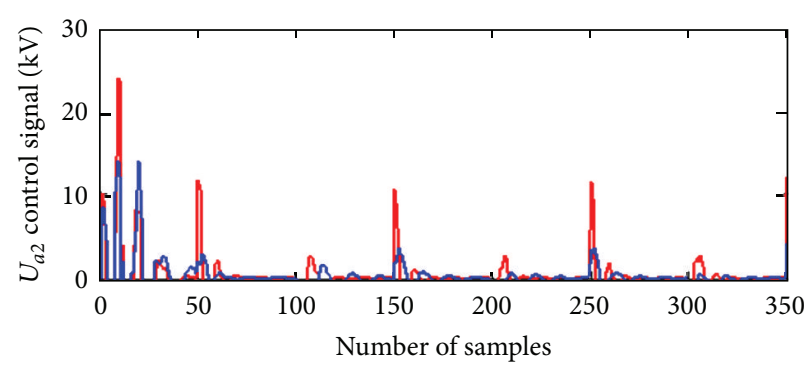

(b)

FIGURE 9: Pickling temperature predictions by GPC (red dots) and DNN-ADNMPC (blue solid) for (a) $T_{2}$ with (b) control signal $U_{a 2}$ to trace the desired reference signal (green solid).

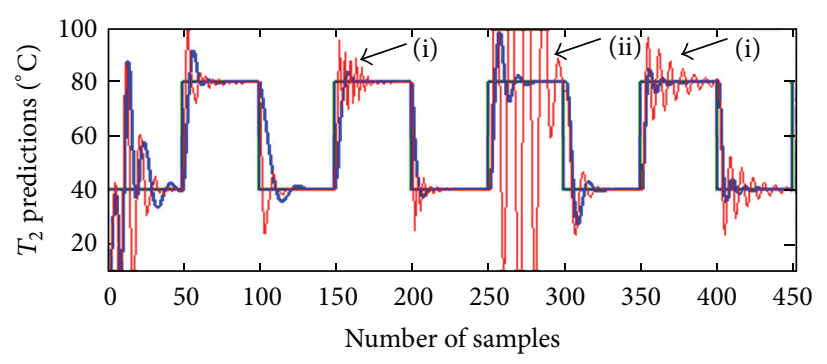

(a)

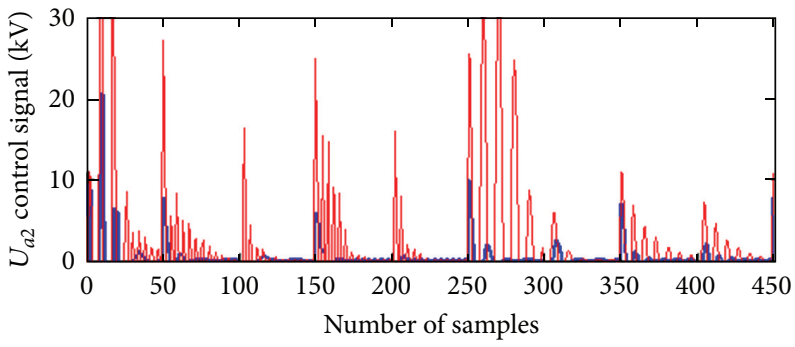

(b)

FIGURE 10: Temperature control in first pickling bath under model mismatch case by GPC (red) and DNN-ADNMPC (blue).

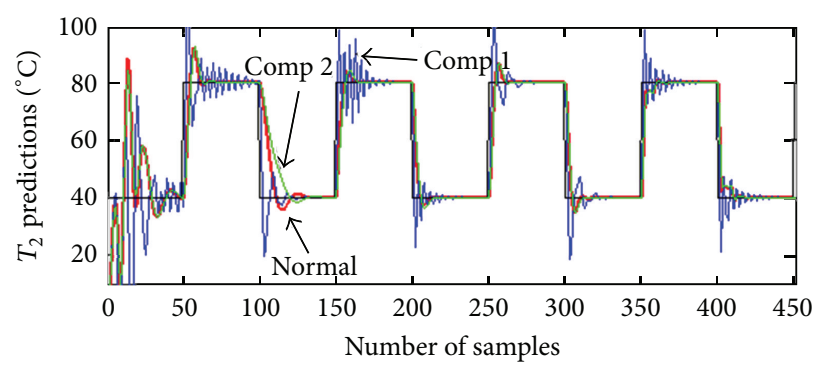

(a)

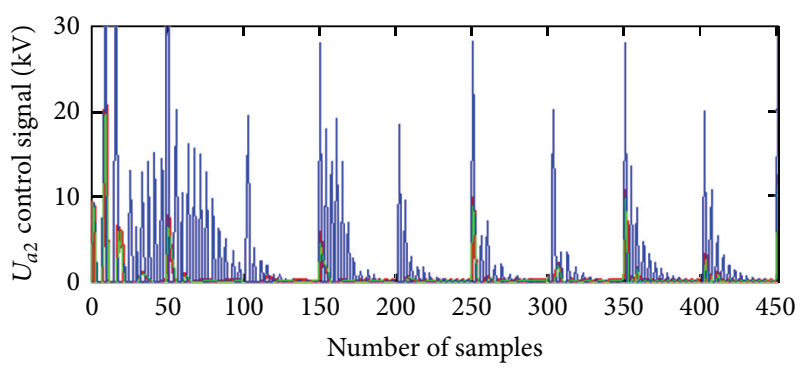

(b)

FIGURE 11: Parameters of DNNADNMPC control strategies (Table 1) affecting performance of temperature control in first pickling bath.

pickling process is 90 seconds while the average computation time identification and control loop using the GPC at each sampling instant is $8.0506 \mathrm{~s}$ while that for the ADNMPC is $15.1025 \mathrm{~s}$. As can be seen in Figures 9 and 10, the identification and control using ADNMPC strategy outperforms the GPC at the expense of extra computational time. The computational time of the ADNMPC is approximately two times when compared to that taken by the GPC. This implies that current personal computer technologies are adequate to solve computationally intensive tasks for system with long sampling time intervals, such as the pickling process, whereas for the systems with faster dynamics and relatively short sampling time intervals, such as thermal runaway in microwave heating, advanced processing technologies must be used to match the constraints.

\section{Conclusions}

Through investigations of dynamical NN-based adaptive model identification and in adaptive direct nonlinear prediction control strategy on microwave heating bath solution for pickling cold-rolled titanium process, the following conclusions can be drawn:

(1) The variable gain, variable forgetting factor, and resetting RLS algorithm training dynamic NN-based identification scheme can adapt to changes in timevarying nonlinear, distribution parameters process under different operating conditions.

(2) The 3-step ahead prediction accuracy and 2 control horizons based control strategy of the adaptive direct 
nonlinear model prediction can perfectly control the temperatures of pickling baths using microwave heating which is the lack of knowledge of dielectric data in the microwave frequency range as a function of temperature.

(3) The simulation results of the identification accuracy and control performance show that the DNNADNMPC outperforms the GPC for microwave heating for pickling cold-rolled titanium process in several cases for set point tracking, model mismatch (model-order change and time-lag change) at the expense of extra computational time.

(4) In the DNN model identification and ADNMPC control, as for long sampling interval, PC can be fitted, while, as for faster dynamics and short sampling interval, the advanced computing technologies must be required.

\section{Nomenclature}

A: $\quad$ Area of operating tank, $\mathrm{m}^{2}$

$C_{i}: \quad$ Specific heat capacity, $\mathrm{J} \mathrm{kg}^{-1} \mathrm{~K}^{-1}$

$C_{\text {ma }}$ : Concentration of mixed acid of $\mathrm{HNO}_{3}$ and $\mathrm{HF}$, $\mathrm{mol} \mathrm{L}^{-1}$

D: The depth of microwave penetration, $\mathrm{m}$

E: Electric field intensity of microwave heating, $\mathrm{V} \mathrm{m}{ }^{-1}$

$E_{\mathrm{ae}}: \quad$ Activation energy, $\mathrm{kJ} \mathrm{mol}^{-1}$

F: $\quad$ Mass flow, $\mathrm{kg} \mathrm{min}^{-1}$

$\Delta H_{R}$ : Heat of reaction per mole of mixed acid and titanium oxides that are reacted, $\mathrm{kJ} \mathrm{mol}^{-1}$

$h$ : Liquid level of operating tank, $\mathrm{m}$

$N$ : The number of data pairs

$n_{m}$ : The number of the magnetrons

$T_{i}$ : Temperature, ${ }^{\circ} \mathrm{C}$

$T: \quad$ The sampling interval, $\mathrm{s}$

$U_{a}: \quad$ Anodic voltage of the magnetron, $\mathrm{kV}$

$I_{a}$ : Anodic current of the magnetron, $\mathrm{mA}$

$R: \quad$ Molar gas constant, $\mathrm{kJ} \mathrm{mol}^{-1} \mathrm{k}^{-1}$

$t$ : Time, min

$e: \quad$ Estimation error

$r$ : Reaction rate constant, $\mathrm{mol} \mathrm{L}^{-1} \mathrm{~min}^{-1}$

$P$ : $\quad$ Microwave power absorption, $\mathrm{W}$

$f$ : $\quad$ Frequency of the incident wave, $2450 \mathrm{MHz}$

$q$ : Amount of bath solution that stuck with cold-rolled titanium, $\mathrm{kg} \mathrm{min}^{-1}$

$k$ : The number of samples

$k_{0}$ : The frequency factor, $\mathrm{min}^{-1}$

$V$ : Volume of operating tank, $\mathrm{m}^{3}$.

\section{Greek Symbols}

$\rho: \quad$ Density of the liquid, $\mathrm{kg} \mathrm{m}^{-3}$

$\phi$ : The electrical energy conversion to microwave efficiency, \%

$\varepsilon(i)$ : The $i$ th tolerance levels

$\sigma$ : Conductivity, $\mathrm{S} \mathrm{m}^{-1}$ $\varepsilon_{0} \varepsilon_{r}^{\prime} \varepsilon_{\text {eff }}^{\prime}$ : Permittivity of the vacuum, of the relative, and of pickling solution, $\mathrm{F} \mathrm{m}^{-1}$

$\lambda_{0}: \quad$ The incident wavelength, $\mathrm{m}$

$\tan \delta$ : The loss tangent

$\eta$ : $\quad$ Delay operator.

Subscripts

$\begin{array}{ll}\text { sp: } & \text { Set point } \\ i: & \text { Number } \\ k: & \text { At time } k \\ k-i: & \text { At time } k-i .\end{array}$

\section{Conflict of Interests}

The authors declare that there is no conflict of interests regarding the publication of this paper.

\section{Acknowledgments}

The authors gratefully acknowledge Professor Jinhui Peng who is the corresponding author. The authors would like to acknowledge the financial support provided under Project (no. KKSY201503006) supported by Scientific Research Foundation of Kunming University of Science and Technology, Yunnan, and Project (no. 51090385) supported by the Major Program of National Natural Science Foundation of China.

\section{References}

[1] W. F. Kladnig, "New development of acid regeneration in steel pickling plants," Journal of Iron and Steel Research, International, vol. 15, no. 4, pp. 1-6, 2008.

[2] B. Yang, J. Peng, S. Guo, S. Zhang, W. Li, and T. He, "Acidpickling plates and strips speed control system by microwave heating based on self-adaptive fuzzy PID algorithm," Journal of Central South University, vol. 19, no. 8, pp. 2179-2186, 2012.

[3] L.-F. Li, P. Caenen, M. Daerden et al., "Mechanism of single and multiple step pickling of 304 stainless steel in acid electrolytes," Corrosion Science, vol. 47, no. 5, pp. 1307-1324, 2005.

[4] M. Regel-Rosocka, "A review on methods of regeneration of spent pickling solutions from steel processing," Journal of Hazardous Materials, vol. 177, no. 1, pp. 57-69, 2010.

[5] B. Tang, W. Su, J. Wang, F. Fu, G. Yu, and J. Zhang, "Minimizing the creation of spent pickling liquors in a pickling process with high-concentration hydrochloric acid solutions: mechanism and evaluation method," Journal of Environmental Management, vol. 98, no. 1, pp. 147-154, 2012.

[6] A. Agrawal and K. K. Sahu, "An overview of the recovery of acid from spent acidic solutions from steel and electroplating industries," Journal of Hazardous Materials, vol. 171, no. 1, pp. 61-75, 2009.

[7] B. Schmidt, R. Wolters, J. Kaplin et al., "Rinse water regeneration in stainless steel pickling," Desalination, vol. 211, no. 1, pp. 64-71, 2007.

[8] J. M. Camp, The Making, Shaping and Treating of Steel, Association of Iron and Steel Engineers, Pittsburgh, Pa, USA, 1995. 
[9] P. Kittisupakorn and P. Kaewpradit, "Integrated data reconciliation with generic model control for the steel pickling process," Korean Journal of Chemical Engineering, vol. 20, no. 6, pp. 985991, 2003.

[10] P. Kittisupakorn, P. Thitiyasook, M. A. Hussain, and W. Daosud, "Neural network based model predictive control for a steel pickling process," Journal of Process Control, vol. 19, no. 4, pp. 579-590, 2009.

[11] W. Daosud, P. Thitiyasook, A. Arpornwichanop, P. Kittisupakorn, and M. A. Hussain, "Neural network inverse modelbased controller for the control of a steel pickling process," Computers and Chemical Engineering, vol. 29, no. 10, pp. 21102119, 2005.

[12] B. Sohlberg, "Hybrid grey box modelling of a pickling process," Control Engineering Practice, vol. 13, no. 9, pp. 1093-1102, 2005.

[13] R. K. Shah, B. Thonon, and D. M. Benforado, "Opportunities for heat exchanger applications in environmental systems," Applied Thermal Engineering, vol. 20, no. 7, pp. 631-650, 2000.

[14] L.-G. Chen and W. Huang, Cold-Rolled Steel Sheet Pickling Process and Equipment, The Metallurgical Industry Press, Beijing, China, 2005.

[15] K. E. Haque, "Microwave energy for mineral treatment processes-a brief review," International Journal of Mineral Processing, vol. 57, no. 1, pp. 1-24, 1999.

[16] B. Yang, W. Li, J.-H. Peng, S.-H. Guo, and S.-M. Zhang, "Microwave energy heating process for cycling fluid medium based on APSO-PID control strategy," in Proceedings of the 2nd International Conference on Advances in Energy Engineering (ICAEE '11), vol. 14, pp. 601-607, Energy Procedia, Bangkok: Thailand, December 2011.

[17] C. A. Vriezinga, S. Sánchez-Pedreño, and J. Grasman, "Thermal runaway in microwave heating: a mathematical analysis," Applied Mathematical Modelling, vol. 26, no. 11, pp. 1029-1038, 2002.

[18] J. Zhu, A. V. Kuznetsov, and K. P. Sandeep, "Mathematical modeling of continuous flow microwave heating of liquids (effects of dielectric properties and design parameters)," International Journal of Thermal Sciences, vol. 46, no. 4, pp. 328-341, 2007.

[19] C. A. Vriezinga, "Thermal runaway and bistability in microwave heated isothermal slabs," Journal of Applied Physics, vol. 79, no. 3, pp. 1779-1783, 1996.

[20] K. S. Narendra and K. Parthasarathy, "Identification and control of dynamical systems using neural networks," IEEE Transactions on Neural Networks, vol. 1, no. 1, pp. 4-27, 1990.

[21] J. T. Spooner, M. Maggiore, R. Ordóñez, and K. M. Passino, Stable Adaptive Control and Estimation for Nonlinear Systems: Neural and Fuzzy Approximator Techniques, John Wiley \& Sons, New York, NY, USA, 2002.

[22] V. A. Akpan and G. D. Hassapis, "Nonlinear model identification and adaptive model predictive control using neural networks," ISA Transactions, vol. 50, no. 2, pp. 177-194, 2011.

[23] M. A. Henson, "Nonlinear model predictive control: current status and future directions," Computers \& Chemical Engineering, vol. 23, no. 2, pp. 187-202, 1998.

[24] U. Yüzgeç, Y. Becerikli, and M. Turker, "Dynamic neuralnetwork-based model-predictive control of an industrial baker's yeast drying process," IEEE Transactions on Neural Networks, vol. 19, no. 7, pp. 1231-1242, 2008.

[25] M. E. Salgado, G. C. Goodwin, and R. H. Middleton, "Modified least squares algorithm incorporating exponential resetting and forgetting," International Journal of Control, vol. 47, no. 2, pp. 477-491, 1988.
[26] Y. Xue and J.-X. Qian, "Modified recursive least squares algorithm with variable parameters and resetting for time-varying system," Chinese Journal of Chemical Engineering, vol. 10, no. 3, pp. 298-303, 2002 (Chinese).

[27] R. Chiong, Intelligent Systems for Automated Learning and Adaptation: Emerging Trends and Applications, chapter 4, Hershey: Information Science Reference, New York, NY, USA, 2010.

[28] M. J. Grimble and A. W. Ordys, "Predictive control for industrial applications," Annual Reviews in Control, vol. 25, no. 3, pp. 13$24,2001$.

[29] D. W. Clarke, C. Mohtadi, and P. S. Tuffs, "Generalized predictive control-part I. The basic algorithm," Automatica, vol. 23, no. 2, pp. 137-148, 1987.

[30] J. Sarangapani, Neural Network Control of Discrete-Time Systems, CRC Press, Boca Raton, Fla, USA, 2006.

[31] A. Antoniou and W.-S. Lu, Practical Optimization: Algorithms and Engineering Applications, Springer, New York, NY, USA, 2007.

[32] L. Liung, System Identification: Theory for the User, PrenticeHall, Upper Saddle River, NJ, USA, 1999.

[33] J. M. Maciejowski, Predictive Control with Constraints, Pearson Education Limited, Addison-Wesley Educational Publishers, London, UK, 2002.

[34] M. J. Grimble and A. W. Ordys, "Predictive control for industrial applications," Annual Reviews in Control, vol. 25, no. 1, pp. 1324, 2001.

[35] C. Sittig, M. Textor, N. D. Spencer, M. Wieland, and P.-H. Vallotton, "Surface characterization of implant materials c.p. $\mathrm{Ti}, \mathrm{Ti}-6 \mathrm{Al}-7 \mathrm{Nb}$ and Ti-6Al-4V with different pretreatments," Journal of Materials Science: Materials in Medicine, vol. 10, no. 1, pp. 35-46, 1999. 

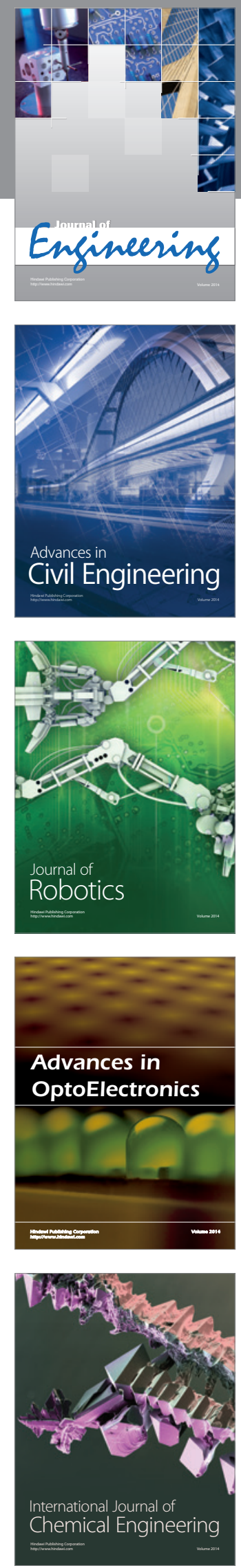

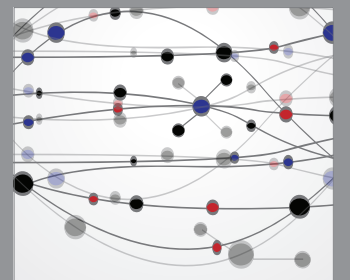

The Scientific World Journal
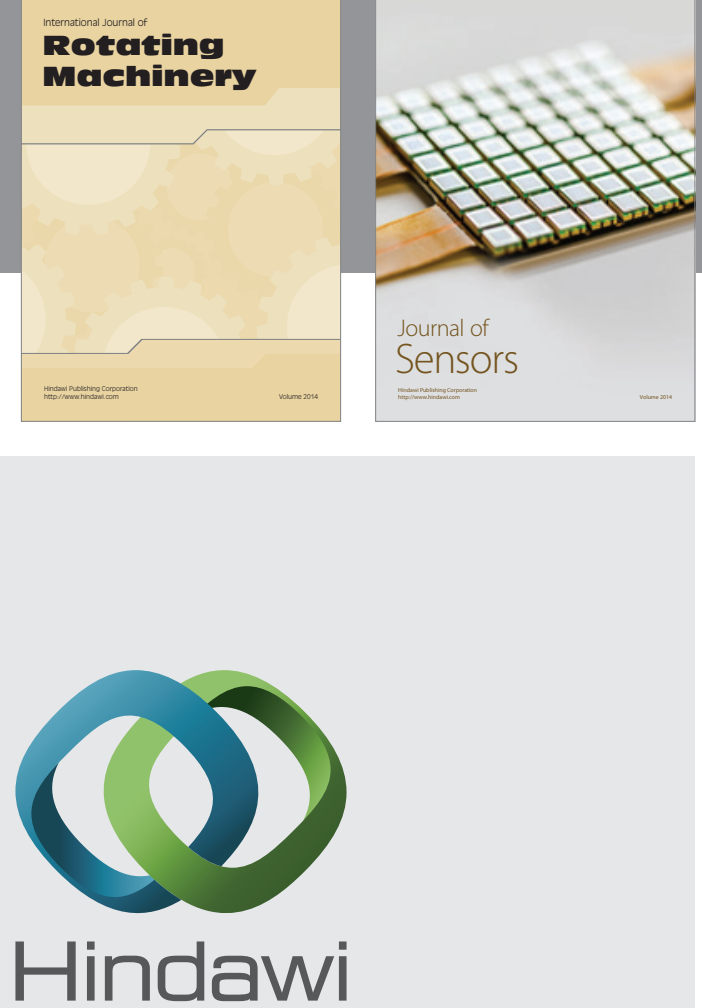

Submit your manuscripts at http://www.hindawi.com
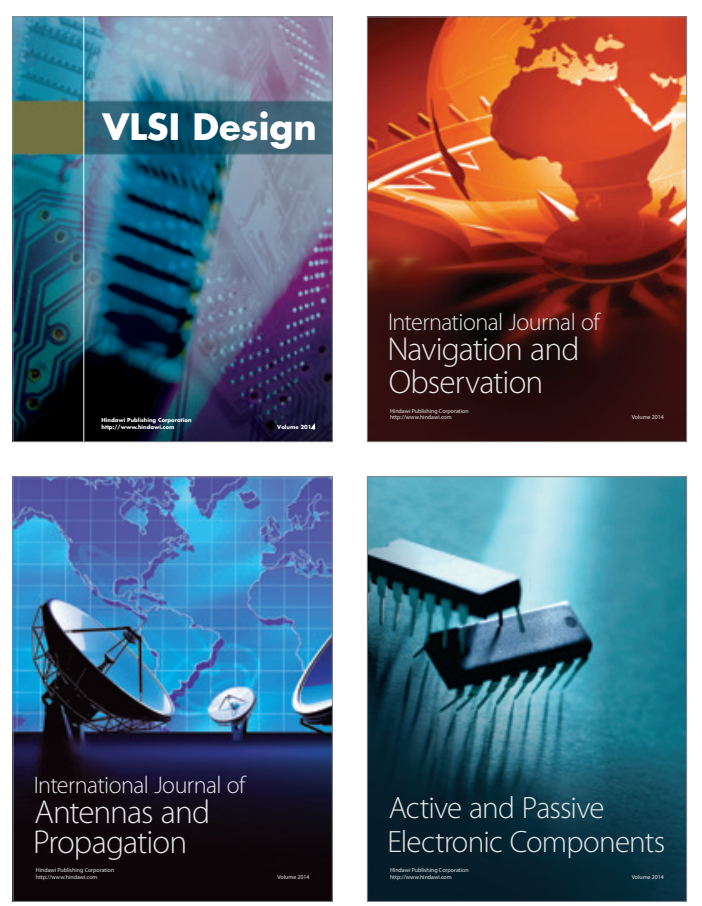
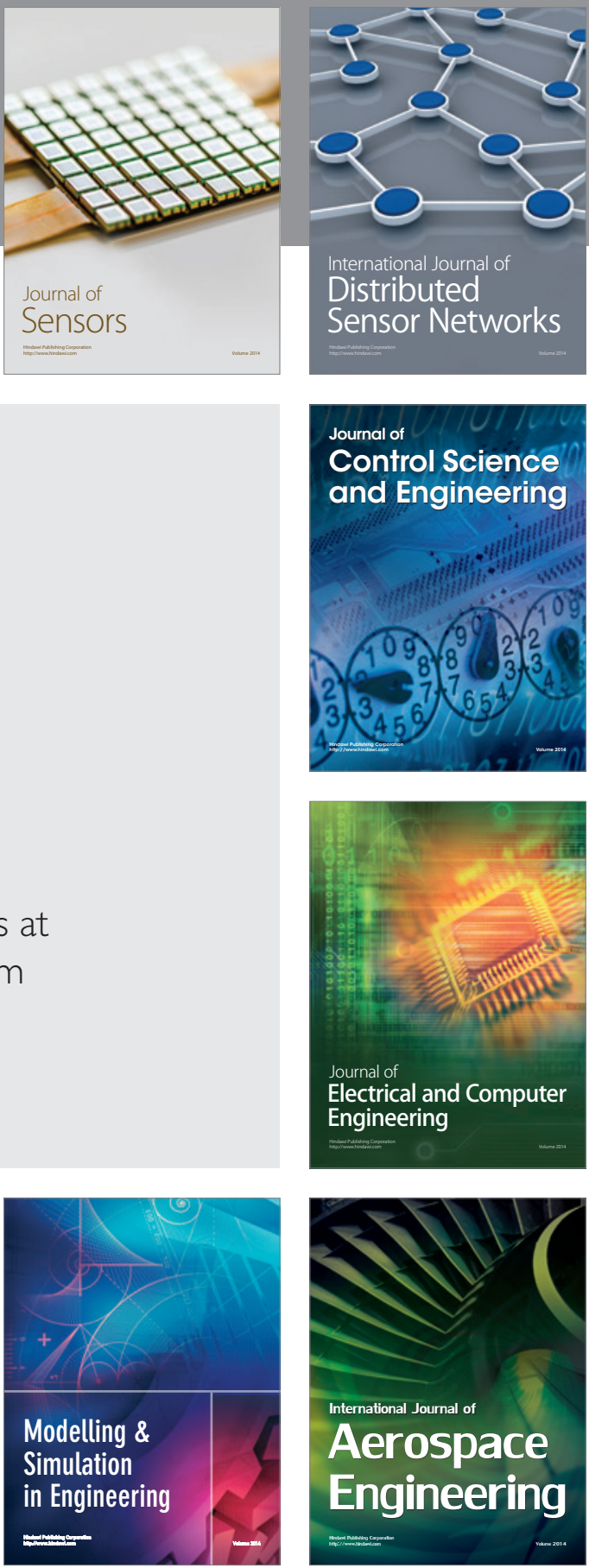

Journal of

Control Science

and Engineering
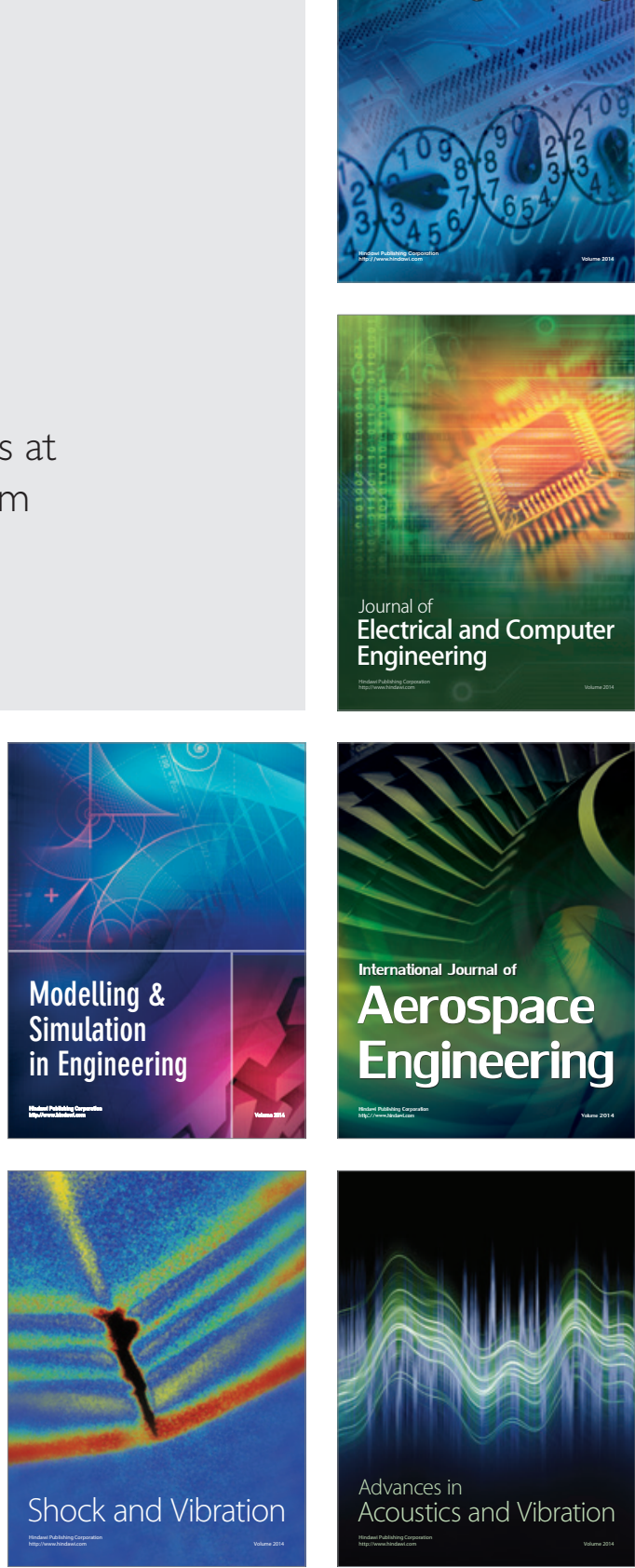